\section{Unlearning fear}

Memory reconsolidation, in which a previously consolidated memory is recalled and updated before subsequent re-storage, may offer an opportunity to attenuate resilient traumatic memories. However, in animal studies, approaches involving behavioural training or pharmacological intervention to attenuate recalled traumatic memories have only been tested on memories of recent traumatic events (for which they have shown some success in modifying). Tsai and colleagues have found that behavioural training is effective at attenuating older ('remote') fear memories in mice if these memories are first epigenetically 'primed' after recall by treatment with a histone deacetylase (HDAC) inhibitor.

To instil traumatic memories in mice, the authors used fear conditioning, in which animals were trained to associate a cue or context (the conditioned stimulus) with a footshock. Repeated exposure led to a freezing response (the fear response) on presentation of the conditioned stimulus alone. To test the effect of targeting reconsolidation to attenuate the fear memory, the authors subjected these mice to fear extinction training 1 day or 30 days later (to examine recent or remote memories, respectively).

Fear extinction training quickly attenuated recent fear memories, and there was no sign of reinstatement of the fear response 30 days later. Remote memories also showed some attenuation immediately after such training; however, cue- or context-triggered fear responses had re-emerged at 30 days, indicating persistence of the original fear memory and suggesting that targeting reconsolidation through behavioural training alone is insufficient to permanently attenuate remote fear memories.

The updating of previously stored memories during reconsolidation is thought to be associated with a period of neuronal plasticity, which may be dependent on gene transcription that is facilitated by acetylation of the underlying chromatin. Chromatin precipitation experiments using hippocampal tissue revealed that after memory recall, mice with remote fear memories (compared with mice with recent fear memories) showed hypoacetylation of the promoter for Fos - an immediate-early gene that is upregulated during neuronal plasticity. Moreover, in contrast to remote memory recall, the recall of recent memories correlated with the inactivation of hippocampal HDAC2 by S-nitrosylation, which promotes chromatin acetylation. These findings suggest that the recall of recent but not remote traumatic memories is associated with a permissive environment for gene transcription that may facilitate neuronal plasticity.

The authors hypothesized that inhibition of HDAC2 may promote attempts to attenuate remote fear memories. Administration of CI-994, which inhibits HDAC2 and other class I HDACs, to fearconditioned mice after memory recall, followed by fear extinction training, resulted in a striking and persistent attenuation of the remote fear memory.

CI-994 treatment followed by fear extinction training was also associated with various changes in the hippocampus, including increased chromatin acetylation, increased expression of various neuronal plasticity-associated genes (including Fos), facilitated long-term potentiation at Schaffer collaterals and an increase in dendritic spine number. These results suggest that HDAC inhibition facilitates remote fear memory attenuation by promoting neuronal plasticity in the hippocampus during reconsolidation.

This study provides a mechanistic basis for why remote fear memories may be so resilient to modification through behavioural therapies, and it offers hope that pharmacological targeting of HDACs in combination with such therapies may successfully attenuate otherwise enduring fear memories.

Darran Yates

ORIGINAL RESEARCH PAPER Gräff, J. et al. Epigenetic priming of memory updating during reconsolidation to attenuate remote fear memories. Cell 156, 261-276 (2014)

\section{in contrast}

to remote

memory

recall, the

recall of recent

memories

correlated with

the inactivation

of hippocampal

HDAC2 by

S-nitrosylation, which

promotes

chromatin

acetylation

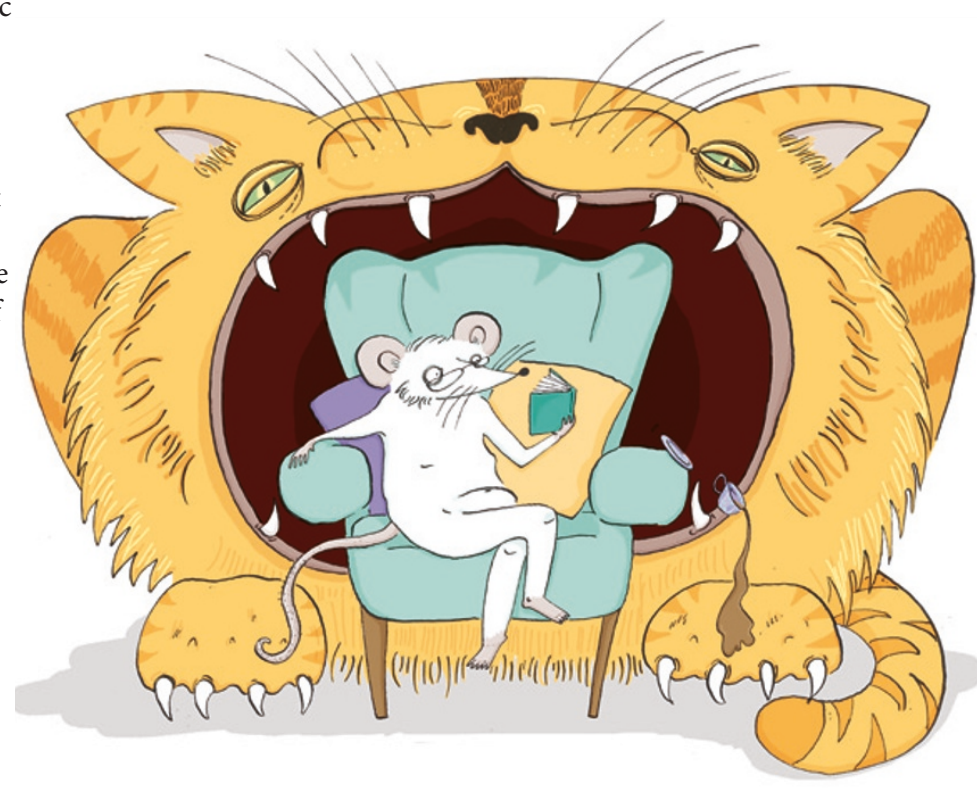

J. Vallis/NPG 